\title{
One-year outcome of bevacizumab therapy for chronic macular edema in central and branch retinal vein occlusions in real-world clinical practice in the UK
}

\author{
This article was published in the following Dove Press journal: \\ Clinical Ophthalmology \\ 25 September 2015 \\ Number of times this article has been viewed
}

\section{Peck Lin Lip' \\ Huzaifa Malick' \\ Kenan Damer' \\ Samer Elsherbiny' \\ Kanupriya M Darrad' \\ Bushra Mushtaq' \\ Arijit Mitra' \\ Panagiota Stavrou' \\ Yit Yang ${ }^{1,2}$}

'Birmingham and Midland Eye Centre, City Hospital, ${ }^{2}$ School of Health and Life Sciences, Aston University, Birmingham, UK
Correspondence: Peck Lin Lip Birmingham and Midland Eye Centre, City Hospital, Dudley Road, Birmingham, BI8 7QH, UK

Tel +44 I2 I507 6808

$\mathrm{Fax}+44$ I2 I507 679।

Email pllipwoo@gmail.com
Background: The purpose of this study was to investigate the 12-month outcome of macular edema secondary to both chronic and new central and branch retinal vein occlusions treated with intravitreal bevacizumab in the real-life clinical setting in the UK.

Methods: Retrospective case notes analysis of consecutive patients with retinal vein occlusions treated with bevacizumab in 2010 to 2012. Outcome measures were visual acuity (measured with Snellen, converted into logMAR [logarithm of the minimum angle of resolution] for statistical calculation) and central retinal thickness at baseline, 4 weeks post-loading phase, and at 1 year.

Results: There were 56 and 100 patients with central and branch retinal vein occlusions, respectively, of whom $62 \%$ had chronic edema and received prior therapies and another $32 \%$ required additional laser treatments post-baseline bevacizumab. Baseline median visual acuity was 0.78 (interquartile range [IQR] 0.48-1.22) in the central group and 0.6 (IQR 0.3-0.78) in the branch group. In both groups, visual improvement was statistically significant from baseline compared to post-loading ( $P<0.001$ and $P=0.03$, respectively), but was not significant by month 12 ( $P=0.058$ and $P=0.166$, respectively); $30 \%$ improved by at least three lines and $44 \%$ improved by at least one line by month 12 . Baseline median central retinal thickness was $449 \mu \mathrm{m}$ (IQR 388-553) in the central group and $441 \mu \mathrm{m}$ (IQR 357-501) in the branch group. However, the mean reduction in thickness was statistically significant at post-loading $(P<0.001)$ and at the 12 -month time point $(P<0.001)$ for both groups. The average number of injections in 1 year was 4.2 in the central group and 3.3 in the branch group.

Conclusion: Our large real-world cohort results indicate that bevacizumab introduced to patients with either new or chronic edema due to retinal vein occlusion can result in resolution of edema and stabilization of vision in the first year.

Keywords: chronic, risk factors, laser, loading dose, subretinal fluid

\section{Introduction}

Retinal vein occlusion is a common cause of visual morbidity in both the elderly and younger populations and can be associated with cardiovascular and other systemic disorders. $^{1-4}$

In recent years, there have been a number of well-designed, randomized clinical trials, demonstrating the efficacy and safety of several therapeutic agents in achieving visual improvement and resolution of macular edema in eyes with central retinal vein occlusion (CRVO) or branch retinal vein occlusion (BRVO). ${ }^{5-11}$ However, prior to the approval of licensed therapy by the National Institute for Health and Care Excellence 
(NICE) for retinal vein occlusion, many ophthalmologists in our National Health Service used intravitreal bevacizumab for treatment of macular edema secondary to CRVO or BRVO. ${ }^{12-14}$ The pathway-orientated approach in our health service provides a good environment for reporting of realworld outcomes, because the criteria used for case selection for initial treatment and decision-making on retreatment is usually very uniform and comprehensive, and free from bias caused by patient factors such as affordability and health insurance coverage. Real-world outcomes are not only helpful for justifying continuation of current clinical practice according to the guidelines derived from clinical trials, but can also provide benchmarks for future clinical audits and possible signals for hypothesis generation in future clinical research.

Numerous real-world outcomes of bevacizumab have been published in the international literature, but to our knowledge, no such large study in the UK National Health Service setting has been published. ${ }^{15-18}$ In this study, we report the findings of the largest comprehensive retrospective audit of all consecutive patients who underwent treatment for macular edema secondary to CRVO or BRVO in a large tertiary center using bevacizumab monotherapy as the first-line agent for retinal vein occlusion therapy or as an additional therapy for chronic macular edema refractory to previous laser therapy or intravitreal triamcinolone therapy.

\section{Materials and methods}

Consecutive patients commenced on treatment with intravitreal bevacizumab for CRVO or BRVO in one eye at a teaching and tertiary referral center from 2010 to 2012 (bevacizumab was first introduced to our department in 2010) were identified from our departmental database. Retrospective data collection from case notes was performed to allow for at least 12 months of follow-up data. This retrospective data review was part of our departmental audit process and hence no ethics approval was needed.

Chronicity of macular edema was defined as persisting disease (macular edema) of more than 12 months' duration and previously receiving laser therapy or intravitreal steroid injections. All patients entering the bevacizumab retinal vein occlusion program followed a uniform pragmatic diagnostic pathway consisting of visual acuity recording using the Snellen chart, slit lamp ophthalmic examination, and optical coherence tomography (OCT) scans on every assessment visit. Central retinal thickness (CRT) was captured and measured (from retinal pigment epithelial layer to retinal surface) using Topcon OCT 2000. The treatment protocol consisted of an intravitreal loading dose of bevacizumab (one to three injections monthly, at the discretion of the treating consultant), followed by repeat injections as clinically indicated if there was persistent or worsening of CRT on follow-up OCT scans. All patients were reviewed 4 weeks post last injection of loading dose, then 4-8 weekly if stable. Data collected from the case notes of each patient included demographics, presence of preexisting glaucoma (including ocular hypertension), bilateral or unilateral treatment, number of injections, visual acuity of treated eyes at baseline, post-loading, and at month 12 , and history of prior laser or intravitreal corticosteroid therapy. OCT scans for all patients were reviewed to collect data on CRT and the presence of subretinal fluid at baseline and at the 1-year time point. Snellen fractions were converted to noncontinuous logMAR (logarithm of the minimum angle of resolution) units by a standard conversion method for statistical purposes. ${ }^{19,20}$

Descriptive statistics were used to show demographics, baseline characteristics, and preexisting risk factors such as glaucoma and cardiovascular status. "Glaucoma" in our series was defined as high intraocular pressure more than $21 \mathrm{mmHg}$ with or without visual field defect, but using at least one topical anti-glaucoma treatment. Data analysis was separately performed for CRVO and BRVO patients to investigate change in visual and anatomical outcomes after initial loading phase and at the 1-year time point. Changes of visual acuity and CRT measurements at these time points from baseline were tested for statistical significance using the Wilcoxon signed-rank test.

\section{Results \\ Demographics and baseline characteristics}

Of 156 patients, 56 had CRVO and 100 had BRVO. All patients had unilateral bevacizumab. All patients completed 12 months of follow-up, but five patients with CRVO were followed up in peripheral units prior to their month 12 visits. In these five patients, final visual acuity at month 12 was obtainable, but their 12-month CRT measurements were not obtainable. For CRVO and BRVO, respectively, the mean age was 69 years and 71 years, and males comprised $46 \%$ and $51 \%$ (Table 1). Baseline median visual acuity in logMAR

Table I Demographic and baseline characteristics

\begin{tabular}{lll}
\hline & CRVO (n=56) & BRVO (n=100) \\
\hline Mean age, years (SD; range) & $69(12.2 ; 34-87)$ & $7 \mid(I I .05 ; 43-94)$ \\
Sex, male/female & $26 / 30$ & $5 I / 49$ \\
Mean number of injections & $4.2(3 ; 1-9)$ & $3.3(3 ; 1-9)$ \\
in I year (mode, range) & & \\
Loading frequency I:2:3 & $13: 9: 34$ & $20: 21: 59$ \\
\hline
\end{tabular}

Abbreviations: CRVO, central retinal vein occlusion; BRVO, branch retinal vein occlusion; SD, standard deviation. 
Table 2 Baseline and changes in vision and central retinal thickness with bevacizumab therapy

\begin{tabular}{|c|c|c|}
\hline & CRVO group & BRVO group \\
\hline \multicolumn{3}{|c|}{ Visual changes in logMAR (Snellen equivalent), compared with baseline } \\
\hline Baseline median VA [IQR] & $0.78(6 / 36)[0.48-1.22]$ & $0.60(6 / 24)[0.30-0.78]$ \\
\hline Median VA post-loading [IQR] & $0.69(6 / 30)[0.18-1.0], P=0.03$ & $0.48(6 / 18)[0.30-0.70], P<0.001$ \\
\hline Median VA at I2 months [IQR] & $0.78(6 / 36)[0.30-1.30], P=0.166$ & $0.54(6 / 20)[0.30-0.78], P=0.058$ \\
\hline Three-line visual improvement at 12 months & $30 \%$ & $24 \%$ \\
\hline One-line visual improvement at 12 months & $44 \%$ & $51 \%$ \\
\hline \multicolumn{3}{|l|}{ CRT changes $(\mu \mathrm{m})$ versus baseline } \\
\hline Baseline median CRT [IQR] & 449 [388-553] & $4 I I[357-50 I]$ \\
\hline Median CRT [IQR] post-loading & 287 [237-402], $P<0.001$ & $309[260-437], P<0.001$ \\
\hline Median CRT [IQR] at I2 months & 278 [229-495], $P<0.001$ & $335[26 I-447], P<0.00 I$ \\
\hline
\end{tabular}

Abbreviations: CRVO, central retinal vein occlusion; BRVO, branch retinal vein occlusion; VA, visual acuity; IQR, interquartile range (statistical analysis using Wilcoxon signed-rank test); CRT, central retinal thickness; logMAR, logarithm of the minimum angle of resolution.

units was 0.78 (Snellen 6/36) and 0.60 (Snellen 6/24) and baseline median CRT was $449 \mu \mathrm{m}$ and $441 \mu \mathrm{m}$ in CRVO and BRVO, respectively (Table 2). The mean number of injections over 12 months was 4.2 and 3.3, respectively, for CRVO and BRVO (Table 1). Prior treatment included laser therapy (namely macular laser and/or pan-retinal photocoagulation as clinically indicated by ischemia) in $45 \%$ of CRVO eyes and $36 \%$ of BRVO eyes, and intravitreal triamcinolone in $20 \%$ of CRVO eyes and $2 \%$ of BRVO eyes. On baseline OCT, the presence of subretinal fluid was found in $57 \%$ of CRVO eyes and $81 \%$ of BRVO eyes.

In terms of preexisting risk factors, we found that a high percentage of patients had glaucoma: 36\% in CRVO group, $14 \%$ in BRVO group; and cardiovascular disease was known in $46 \%$ of CRVO and $36 \%$ of BRVO patients (Table 3 ).

\section{Visual outcome}

At the post-loading review, in CRVO eyes, median visual acuity was logMAR 0.69 (interquartile range [IQR] 0.18-1.0), but was reduced to 0.78 (IQR $0.3-1.3$ ) at the month 12 visit (Table 2). The improvement from baseline to the post-loading visit was statistically significant $(P=0.03)$, but the improvement from baseline to month 12 was not significant $(P=0.166)$. In terms of proportion of patients improving, $30 \%$ improved by at least three lines and $44 \%$ improved by at least one line by month 12 .

Similarly, in BRVO eyes at post-loading review, median visual acuity was $6 / 18$ or $\log$ MAR 0.48 (IQR $0.3-0.7$ ), but was 6/20 or logMAR 0.54 (IQR $0.3-0.78$ ) at the month 12 visit. The improvement from baseline to the post-loading visit was significant $(P<0.001)$, but the improvement from baseline to month 12 was not significant $(P=0.058)$. In terms of proportions of patients improving, $24 \%$ improved by at least three lines and 51\% improved by at least one line by month 12 .

\section{Anatomical outcomes}

Based on a total of 51 CRVO eyes with a complete CRT dataset, median CRT improved to $287 \mu \mathrm{m}$ (IQR 237-402) post-loading, and to $278 \mu \mathrm{m}$ (IQR 229-495) at month 12 (Table 2). Median reduction in CRT from baseline was statistically significant at the post-loading visit $(P<0.001)$ as well as at the month 12 visit $(P<0.001)$.

Table 3 Preexisting risk factors, previous therapy, and adverse effects

\begin{tabular}{|c|c|c|}
\hline & CRVO $(n=56)$ & BRVO $(n=100)$ \\
\hline \multicolumn{3}{|l|}{ Recorded risk factors } \\
\hline Known cardiovascular risks & $26(46 \%)$ & $36(36 \%)$ \\
\hline Referral to cardiovascular assessment (recorded in notes) & $10(18 \%)$ & $10(10 \%)$ \\
\hline Known glaucoma/ocular hypertension & $20(36 \%)$ & $14(14 \%)$ \\
\hline \multicolumn{3}{|l|}{ Chronicity indication } \\
\hline Had previous laser therapy & $25(45 \%)$ & $36(36 \%)$ \\
\hline Had previous triamcinolone & II (20\%) & $2(2 \%)$ \\
\hline \multicolumn{3}{|l|}{ Adverse effects } \\
\hline \multirow[t]{2}{*}{ Raised IOP } & $2(4 \%)$ & $5(5 \%)$ \\
\hline & I (rubeotic glaucoma) & \\
\hline Corneal abrasion & 0 & $2(2 \%)$ \\
\hline Endophthalmitis & 0 & 0 \\
\hline
\end{tabular}

Abbreviations: CRVO, central retinal vein occlusion; BRVO, branch retinal vein occlusion; IOP, intraocular pressure. 
In the BRVO eyes, median CRT improved to $309 \mu \mathrm{m}$ (IQR 260-437) post-loading and to $335 \mu \mathrm{m}$ (IQR 261-447) at month 12. The median change in CRT from baseline was statistically significant at the post-loading visit $(P<0.001)$ and at month $12(P<0.001)$.

Thirty-two of $56(57 \%)$ CRVO eyes that had subretinal fluid at baseline achieved a mean $\log$ MAR visual acuity of 0.84 (Snellen equivalent of 6/40) at month 12 . This was numerically slightly worse compared to that achieved by the whole cohort of CRVO eyes. Eighty-one of 100 (81\%) BRVO eyes that had subretinal fluid at baseline achieved a median logMAR visual acuity of 0.55 (Snellen equivalent of 6/20) at month 12 . This was numerically similar to that achieved by the whole cohort of BRVO eyes.

\section{Adverse events}

One patient developed mild ocular hypertension and another progressed to rubeotic glaucoma in the CRVO group. In the BRVO group, five patients had mild controlled ocular hypertension and two patients had corneal abrasion. There was no endophthalmitis in our series (Table 3).

\section{Discussion}

Since 2008, prospective studies of bevacizumab have provided the necessary evidence base for ophthalmologists worldwide to use such an unlicensed therapy for retinal vein occlusion. ${ }^{15,18}$ In many centers, including ours, prior to NICE approval of ranibizumab for retina vein occlusion in 2013, ${ }^{13}$ bevacizumab was used as the anti-vascular endothelial growth factor (VEGF) agent of choice for patients with retinal vein occlusion. Although there are fewer real-world studies of retinal vein occlusion using bevacizumab than ranibizumab, it is still a challenge to make a limited comparison of small numbers. For example, more recently, the MARVEL group published a prospective, head-to-head study comparing bevacizumab with ranibizumab for BRVO. ${ }^{21}$ Although the numbers are small, the initial 6-month result suggested a compatible benefit of either agent in treating BRVO with macular edema using the "as required regime", with an average of three to four injections needed in the first 6 months. In a study from Copenhagen, the authors reported real-world outcomes for 106 patients with retinal vein occlusion treated with ranibizumab, whereas in another study from Rome, 50 eyes with CRVO were included..$^{22,23}$

Thirty percentage of CRVO eyes improved by at least three lines and $44 \%$ improved by at least one line by month 12. This was very similar to the proportion found in the Copenhagen study, although slightly less than that found in the CRUISE study, which reported up to $47 \%$ with a threeline gain at month 6 and $50.8 \%$ by month 12 . In BRVO eyes, we found that $24 \%$ improved by at least three lines and $51 \%$ improved by at least one line by month $12 . .^{5,6}$ This was almost identical to the findings from the Copenhagen study, but again very much less than that reported in the BRAVO study (up to $60.3 \%$ with a three-line gain by month 12 ). ${ }^{22}$ Reasons for the lower visual outcome may be attributable to the fewer injections in our population (mean 4.2 CRVO and 3.3 BRVO in 12 months) compared with 9.3 injections in 12 months reported in the extended CRUISE and BRAVO trial. ${ }^{7}$ Other reasons may be the lack of refraction on every visit and our reliance on OCT retreatment criteria rather than a low retreatment threshold using both functional and anatomical criteria as used in the CRUISE and BRAVO studies. ${ }^{5,6}$ The change could also be due to a true difference between ranibizumab and bevacizumab, although this seems unlikely, given the findings in head-to-head studies on other indications, such as age-related macular degeneration and diabetic macular edema. ${ }^{24-30}$

Our series had a variable number of injections in the loading phase depending on clinician discretion, and therefore had an overall lower number of injections over a 12-month period. Despite the much lower injection rates in both CRVO and BRVO eyes, we found that CRT measurements were significantly reduced and maintained at month 12 . In this respect, our findings compare favorably with the CRUISE, BRAVO extension study (HORIZON), which followed patients for up to 2.5 years in total from their first injections. ${ }^{7}$ This suggests that an objective parameter such as CRT may be a suitable and indeed practical method to be adopted in real-world practices in the UK. The achievement of a good outcome in CRT with much fewer injections suggests that use of anatomical retreatment criteria may not only be more pragmatic, but may also result in reducing possible unnecessary retreatment. As a result of this study, since the approval of ranibizumab for retinal vein occlusion at our center, we have continued to use OCT-driven criteria where we repeat ranibizumab dosing if there is persistent or worsening macular edema on an asneeded basis after a variable loading of one to three doses, depending on the severity of edema at baseline.

Adverse events were infrequent, highlighting the wellestablished safety profile of bevacizumab in this population. Notably, only one patient had rubeotic glaucoma after commencing on bevacizumab therapy. This is a recognized complication of ischemic retinal vein occlusion, but has not been reported specifically in eyes undergoing treatment with intravitreal bevacizumab or ranibizumab. ${ }^{31}$ This highlights 
the need to treat peripheral retinal non-perfusion, especially in cases of CRVO when discontinuing injections after successful resolution of macular edema.

It is well recognized that up to $60 \%$ of patients with retinal vein occlusions have systemic hypertension or cardiovascular disease. ${ }^{4}$ In our retrospective series, which relied on extracting data from clinical notes, we found considerably fewer patients with associated cardiovascular conditions. We believe this may have been due to poor documentation by clinicians and also a tendency by ophthalmologists to ignore systemic factors when treating macular edema. Given the $5 \%-10 \%$ risk of retinal vein occlusion developing in the second eye within a 2-3-year period, it is important for us to continually highlight the importance of cardiovascular screening and management in this population of patients. ${ }^{1-4}$

In common with real-world studies, the major weakness of this study was the lack of protocol refraction and visual acuity measurements using research standard logMAR visual acuity charts. Visual acuity comparisons were therefore made using the Wilcoxon signed-rank test, which was appropriate for nonparametric data. Although we used a standard method of converting Snellen visual acuity to $\log$ MAR units, there is a known tendency to overestimate visual acuity using the Snellen chart at lower levels of acuity, and this may have occurred in our study. However, the strengths of this study were the larger numbers and the high completion rate with OCT parameters at 1 year, allowing us to compare the treatment effect with other studies and landmark clinical trial outcomes with reasonable confidence.

\section{Conclusion}

In this report, we have described our approach to bevacizumab therapy for retinal vein occlusion and also reported our outcomes. We have reinforced the justification to use bevacizumab, especially if other anti-VEGF agents are unavailable. Such evidence from real-world experience may be useful as a pragmatic benchmark in future audits on outcomes of retinal vein occlusion therapy using either bevacizumab or other anti-VEGF agents.

\section{Disclosure}

The authors report no conflicts of interest in this work.

\section{References}

1. McIntosh RL, Rogers SL, Lim L, et al. Natural history of central retinal vein occlusion: an evidence-based systematic review. Ophthalmology. 2010;117(6):1113-1123.e15.

2. Mitchell P, Smith W, Chang A. Prevalence and associations of retinal vein occlusion in Australia. The Blue Mountains Eye Study. Arch Ophthalmol. 1996;114:1243-1247.
3. Rogers S, McIntosh RL, Cheung N, et al. The prevalence of retinal vein occlusion: pooled data from population studies from the United States, Europe, Asia, and Australia. Ophthalmology. 2010;117(2):313-319.

4. The Royal College of Ophthalmology. Interim guidelines for management of retinal vein occlusion. Available from: https://www.rcophth. ac.uk/wp-content/uploads/2014/12/2010-SCI-095-RVO-InterimGuidelines-Dec-2010-FINAL.pdf. Accessed July 17, 2015.

5. Brown DM, Campochiaro PA, Singh RP, et al; CRUISE Investigators. Ranibizumab for macular edema following central retinal vein occlusion: six-month primary end point results of a phase III study. Ophthalmology. 2010;117(6):1124-1133.

6. Varma R, Bressler NM, Suñer I, et al; BRAVO and CRUISE Study Groups. Improved vision-related function after ranibizumab for macular edema after retinal vein occlusion: results from the BRAVO and CRUISE trials. Ophthalmology. 2012;119(10):2108-2118.

7. Heier JS, Campochiaro PA, Yau L, et al. Ranibizumab for macular edema due to retinal vein occlusions: long-term follow-up in the HORIZON trial. Ophthalmology. 2012;119(4):802-809.

8. Campochiaro PA, Sophie R, Pearlman J, et al; RETAIN Study Group. Longterm outcomes in patients with retinal vein occlusion treated with ranibizumab: the RETAIN study. Ophthalmology. 2014;121(1):209-219.

9. Ogura Y, Roider J, Korobelnik JF, et al; GALILEO Study Group. Intravitreal aflibercept for macular edema secondary to central retinal vein occlusion: 18-month results of the phase 3 GALILEO study. Am J Ophthalmol. 2014;158(5):1032-1038.

10. Heier JS, Clark WL, Boyer DS, et al. Intravitreal aflibercept injection for macular edema due to central retinal vein occlusion: two-year results from the COPERNICUS study. Ophthalmology. 2014;121(7): 1414-1420.

11. Haller JA, Bandello F, Belfort R Jr, et al; Ozurdex GENEVA Study Group. Dexamethasone intravitreal implant in patients with macular edema related to branch or central retinal vein occlusion twelve-month study results. Ophthalmology. 2011;118(12):2453-2460.

12. National Institute for Health and Care Excellence. Dexamethasone intravitreal implant for the treatment of macular oedema secondary to retinal vein occlusion. NICE technology appraisal guidance. TA229, July 2011. Available from: https://www.nice.org.uk/guidance/ta229. Accessed July 17, 2015.

13. National Institute for Health and Care Excellence. Ranibizumab for treating visual impairment caused by macular oedema secondary to retinal vein occlusion. NICE technology appraisal guidance. TA283, May 2013. Available from: https://www.nice.org.uk/guidance/ta283. Accessed July 17, 2015.

14. National Institute for Health and Care Excellence. Aflibercept for treating visual impairment caused by macular oedema secondary to central retinal vein occlusion. NICE technology appraisal guidance. TA305, February 2014. Available from: https://www.nice.org.uk/guidance/ ta305. Accessed July 17, 2015.

15. Wu L, Martínez-Castellanos MA, Quiroz-Mercado H, et al; Pan American Collaborative Retina Group (PACORES). Twelve-month safety of intravitreal injections of bevacizumab (Avastin): results of the Pan-American Collaborative Retina Study Group (PACORES). Graefes Arch Clin Exp Ophthalmol. 2008;246(1):81-87.

16. Rabena MD, Pieramici DJ, Castellarin AA, Nasir MA, Avery RL. Intravitreal bevacizumab (Avastin) in the treatment of macular edema secondary to branch retinal vein occlusion. Retina. 2007;27(4):419-425.

17. Epstein DL, Algvere PV, von Wendt G, Seregard S, Kvanta A. Benefit from bevacizumab for macular edema in central retinal vein occlusion: twelve-month results of a prospective, randomized study. Ophthalmology. 2012;119(12):2587-2591.

18. Hikichi T, Higuchi M, Matsushita T, et al. Two-year outcomes of intravitreal bevacizumab therapy for macular oedema secondary to branch retinal vein occlusion. Br J Ophthalmol. 2014;98(2):195-199.

19. Gregori NZ, Feuer W, Rosenfeld PJ. Novel method for analyzing Snellen visual acuity measurements. Retina. 2010;30(7):1046-1050.

20. Snellen - logMAR Visual Acuity Calculator. Available from: http:// www.myvisiontest.com/logmar.php 
21. Narayanan R, Panchal B, Das T, Chhablani J, Jalali S, Ali MH; MARVEL Study Group. A randomised, double-masked, controlled study of the efficacy and safety of intravitreal bevacizumab versus ranibizumab in the treatment of macular oedema due to branch retinal vein occlusion: MARVEL Report No. 1. Br J Ophthalmol. January 28, 2015.

22. Brynskov T, Kemp H, Sørensen TL. Intravitreal ranibizumab for retinal vein occlusion through 1 year in clinical practice. Retina. 2014;34(8): $1637-1643$

23. Pacella E, Pacella F, La Torre G, et al. Testing the effectiveness of intravitreal ranibizumab during 12 months of follow-up in venous occlusion treatment. Clin Ther. 2012;163(6):e413-e422.

24. Chakravarthy U, Harding SP, Rogers CA, et al; IVAN Study Investigators. Alternative treatments to inhibit VEGF in age-related choroidal neovascularization: 2-year findings of the IVAN randomized controlled trial. Lancet. 2013;382(9900):1258-1267.

25. Comparison of Age-related Macular Degeneration Treatments Trials (CATT) Research Group, Martin DF, Maguire MG, Fine SL, et al. Ranibizumab and bevacizumab for treatment of neovascular age-related macular degeneration: two-year results. Ophthalmology. 2012;119(7): $1388-1398$.

26. Ruiz-Moreno JM, Arias L, Montero JA, Carneiro A, Silva R. Intravitreal anti-VEGF therapy for choroidal neovascularization secondary to pathological myopia: 4-year outcome (with CATT and IVAN). Br J Ophthalmol. 2013;97(11):1447-1450.
27. Nepomuceno AB, Takaki E, Paes de Almeida FP, et al. A prospective randomized trial of intravitreal bevacizumab versus ranibizumab for the management of diabetic macular edema. Am J Ophthalmol. 2013; 156(3):502-510.e2.

28. Ford JA, Elders A, Shyangdan D, Royle P, Waugh N. The relative clinical effectiveness of ranibizumab and bevacizumab in diabetic macular oedema: an indirect comparison in a systematic review (with IVAN and CATT). BMJ. 2012;345:e5182.

29. Retinopathy Clinical Research Network, Wells JA, Glassman AR, Ayala AR, et al. Comparative effectiveness randomized clinical trial of aflibercept, bevacizumab, or ranibizumab for diabetic macular edema, protocol T. N Engl J Med. 2015;372:1193-1203.

30. Berg K, Pedersen TR, Sandvik L, Bragadóttir R. Comparison of ranibizumab and bevacizumab for neovascular age-related macular degeneration according to LUCAS treat-and-extend protocol. Ophthalmology. 2015;122(1):146-152.

31. DeCroos FC, Todorich B, Alshareef R, et al. Neovascular events in eyes with central retinal vein occlusion undergoing serial bevacizumab or ranibizumab intravitreal injections: a retrospective review. JOphthalmic Vis Res. 2014;9(4):461-468.
Clinical Ophthalmology

\section{Publish your work in this journal}

Clinical Ophthalmology is an international, peer-reviewed journal covering all subspecialties within ophthalmology. Key topics include: Optometry; Visual science; Pharmacology and drug therapy in eye diseases; Basic Sciences; Primary and Secondary eye care; Patient Safety and Quality of Care Improvements. This journal is indexed on

\section{Dovepress}

PubMed Central and CAS, and is the official journal of The Society of Clinical Ophthalmology (SCO). The manuscript management system is completely online and includes a very quick and fair peer-review system, which is all easy to use. Visit http://www.dovepress.com/ testimonials.php to read real quotes from published authors. 